\title{
Selected Stakeholders' Perceived Supports Needed for Inclusive Education in the Schools of Maldives
}

\author{
Fathmath Nishan \\ Universiti Brunei Darussalam, Brunei
}

\begin{abstract}
In the Maldives, the implementation of inclusive education formally began in 2013 with the inclusive education policy by the Ministry of Education (MoE). It can be very challenging for a country and its schools to implement an advanced inclusive policy over a short period of time [1]. Investigating selected stakeholders' perceived supports for inclusive education can yield vital results and contribute in managing resources for successful implementation of inclusive education in the Maldives. Being the first large scale study on inclusion in the Maldives, this study of a mixed-methods design investigated the perceived supports required by selected stakeholders, using a survey questionnaire and semistructured interviews. The respondents of the survey $(N=170)$ were 125 regular primary teachers, 11 Deputy Principals and 34 SEN teachers from 11 government inclusive schools from 6 different locations in the Republic of Maldives. The data was supplemented with semi-structured interviews of 8 regular teachers, 6 SEN teachers and 5 Deputy Principals. The supports required were teaching materials, teaching assistants, trained teachers, support from school management, and reduced class size. Key recommendations were providing teaching resources, maintaining a reasonable teacher-student ratio, providing teacher support and teacher training.
\end{abstract}

\section{Introduction}

Over the years, many studies have highlighted the benefits of inclusive education [2,3,4,5], emphasizing the fact that opportunities for students with disabilities to interact with their peers in regular classrooms have resulted in positive behaviours and social development. While global movements towards inclusive education occurred, the Maldives also adopted changes in its education system according to inclusive education policy in 2013. However, several challenges in providing education for students with Special Educational Needs (SEN) were faced in the early stages of moving towards inclusive education. One of the main reasons was that the Maldives did not have enough trained teachers for inclusive education [6].
The paradigm shift from special education to inclusive education in the Maldives requires further investigation of theoretical and field research for identifying the perceptions of inclusive education, supports and the challenges encountered in the implementation process. Further, across the globe in the past, students with diverse needs were excluded from public schools. Hence, students with disabilities were not included in the general education system. To address this issue, many legislations came into effect to provide students with diverse needs with equal opportunities for education.

\section{Background to the study}

Although Maldives had taken several initiatives towards special and inclusive education, over the years there had not been a special school established. While there is limited research conducted to investigate the status of the progress of inclusive education in the Maldives, a case study by Naseer in 2012 was able to identify the main obstacles faced by teachers when implementing inclusive education [7]. This study is an indepth study investigating the supports required for inclusive education as perceived by selected deputy principals, regular teachers and SEN teachers in the Maldives. The participants in this study were from 11 government schools from Male', the capital of Maldives, and from other islands which accounted for high number of enrolled students with special needs. The medium of teaching in all these schools were in English Language, except for subjects such as Islam (religious studies), Dhivehi (local language and literature) and Quran. The national curriculum is inclined to prepare students for international exams such as the Cambridge International Exams (CIE), International General Certificate of Education (IGCSE) after completing the 10th grade, and London Examinations: Edexcel International (A' level), by the end of 12 th grade.

In the Maldives, primary schooling and secondary schooling are not separate from each other, even though this was practiced several years ago. At present, the schools in the Maldives offer primary and secondary level education in the same schools. The school statistics for the Republic for the year 2017 show a total gross enrolment of 71588 students [8]. 


\section{Literature Review}

\subsection{Inclusive Education Policy}

The Maldives has always placed special emphasis on access to equal education for all. The Ministry of Education (MoE) is the government body responsible for the provision of education in the Maldives. Similar to the government schools, the private sector also plays a major role in education, providing services from early childhood education tocollege education.

The National Institute of Education (NIE) has been leading the efforts in putting the disability law into practice, their focus being SEN and their longterm vision of inclusive education. As a result of the initiatives and vision of NIE, coupled with the development goals of $\mathrm{MoE}$, the inclusive education policy was implemented on 20th January 2013.

The policy state that all government schools should adopt inclusive education, and provided guidelines for necessary modifications to teaching, in addition to the methods, facilities and responsibilities of the stakeholders to provide formal education to students with disabilities and additional learning supports [8]. The primary principles of the policy were threefold.

Firstly, the policy's focus is to provide equal opportunities within the formal education system for all children since education is the right of all children. Secondly, the policy is a means to establish an adaptive system to include all children as far as possible based on their capabilities in all educationrelated matters, acknowledging their thoughts, ideas and feelings. Thirdly, the policy intends to modify teaching methods to achieve the national criteria objectives, which include all children, and to establish an education system with relevant equipment and resources. Additionally, the policy's focus is on three main categories of children, which are: (1) children who need additional support; (2) children with disabilities; and (3) children under special circumstances [8]. The policy defined children who need additional support as those needing further support and equipment, such as children with learning disabilities; for example, dyslexia, dysgraphia, dyscalculia, gifted and talented and children with Attention Deficit Hyperactivity Disorder (ADHD) and Autism Spectrum Disorder (ASD). The policy defined children with disabilities as children unable to function independently due to physical, mental or health conditions. Children with special needs were categorized as children with impairments such as physical, sensory, hearing, visual, speech, intellectual, and autism. These categories were further classified into degrees such as mild, moderate, severe and profound [8].

\subsection{School Supports for Inclusive Education}

School administrators have a crucial role in the successful implementation of inclusive education. School leaders need to create a collaborative culture and collaborative learning communities. The leaders should also provide instructions for the staff in relation to reaching a shared vision in the school. Additionally, there are other supports the school can offer for teachers and students with SEN. One of the most important things to begin with is accessibility. The school leaders should ensure that the students with SEN have access to all facilities such as classrooms, lavatories, libraries and the play area. Most of the buildings in primary schools in the Maldives have stairs which limits the mobility of students with physical and visual disabilities.

\subsubsection{Teacher training for Inclusive Education.}

Much of the research suggests that teachers do not find themselves to be prepared for inclusive education. In order to scaffold this inadequacy, schools need to provide effective professional development courses or sessions to instill confidence and competence in the general teachers. It was found that most of the teachers reported on their lack of preparation for effective inclusive teaching for diverse populations from their initial teacher training programs [9]. The teachers require systematic and intensive training that includes research-based best practices in inclusive schools to be confident and competent [10]. In a survey it was found that the development of positive attitudes toward inclusion is dependent upon the teacher's professional development [11]. Similarly, the researchers found that $44 \%$ of the surveyed teachers believed that they did not receive enough staff development to prepare them to teach students with special needs [12].

For inclusive education to be successful, the school leaders should ensure that there is adequate number of staff in their schools and that the appropriate technology is provided for the staff [13]. The NCSE policy advice paper in 2013 stated that, to meet the educational needs of students with SEN, the students require qualified teachers equipped with appropriate skills to be competent at teaching students with diverse needs and mainstream teachers needed appropriate training [14]. These findings show that the school administrators have an important role in providing professional development to equip teachers with the knowledge and skills for inclusive education.

\section{Methodology}

\subsection{Research Design}

This study employed a concurrent mixed method design to collect and integrate quantitative and qualitative data for a more comprehensive understanding of the phenomena under investigation.

\subsection{Instruments}

In this study the quantitative data was gathered 
from a larger population of participants in inclusive schools by administering a survey questionnaire. Using a survey questionnaire was the most costeffective and favorable way to gain data from teachers in inclusive schools in the islands due to the geographical setting of the Maldives. To gain the participants' unique perspectives and experiences, a semi-structured interview protocol was used. This enabled the researcher to gain firsthand, first-person account of the participants. Hence, to gather valuable data to provide a greater understanding of the research topic, a mixed methods approach was used in this study.

The participants in this study were all local regular teachers, SEN teachers and deputy principals who worked in government inclusive schools and were involved in the teaching-learning process of students in Key Stage 1 (primary grades 1 to 3 ) and Key Stage 2 (primary grades 4 and 5). Following the sampling procedure, the participants randomly selected for the survey questionnaire were 125 regular teachers (RTs), 34 SEN teachers (STs) and 11 Deputy Principals (DPs). The participants selected purposively for the semi-structured interviews were 8 RTs, 6 STs and 5 DPs.

\subsection{Data Analysis}

The quantitative data from the survey questionnaire was analyzed using SPSS version 23, and the qualitative data of the study was analyzed thematically following the procedure outlined by Braun and Clarke [15].

\section{Findings}

Two main themes emerged for the supports required for inclusive education as perceived by the selected stakeholders of this study, which were resources and school supports.

\subsection{Resources}

One of the perceived support methods needed by the participants for the implementation of inclusive education was resources, with sub-themes; teaching materials, teaching assistants and trained teachers.

5.1.1.Teaching materials. All three groups of teachers, 70 RTs (27.0\%), 23 STs (33.8\%) and 11 DPs $(36.7 \%)$, reported that more teaching materials were required to implement inclusive education. The main resource articulated by the participants was an adapted curriculum.

The teachers were in need of materials for the different levels of learners, such as books, puzzles, worksheets and other educational toys. Teachers reported materials were not available because of budget constraints, time consuming protocols and procedures. Meanwhile, regular teachers who did not have specialized training for inclusive education attempted and prepared worksheets and contributed to purchasing items for students with SEN. This did not suffice.

Similarly, STs also described their difficulties faced as a result of having limited materials. For example, the STs recounted the lack of student interest and engagement in learning with the materials that the students with SEN were too familiar with. Hence, the scarcity of teaching materials posed a barrier for differentiated instruction.

5.1.2. Teaching Assistants. A total of 41 RTs (15.8\%), 3 STs $(4.4 \%)$, and 6 DPs $(20.0 \%)$ disclosed that teaching assistants or shadow teachers were needed in the regular classrooms as a support. Specifically, the RTs voiced that the support of an assistant teacher was required in the regular classroom to help the RTs teach different ability students.

Deputy principals reported the need for teaching assistants. DP4 revealed that School C did not have teachers working as teaching assistants. The RTs were the teachers available to help each other, aside from the school counsellor. According to DP4, School C did not have a group of teachers committed to students with SEN only. However, those teachers played the role of teaching assistants to help the RTs. One concern is the fact the RTs or other RTs helping as teaching assistants were not specially trained for inclusive education. However, the STs believed that the RTs had enough knowledge to cope with students with SEN in a regular classroom.

According to ST4, the issue was not the lack of teaching assistants in the regular classrooms. ST4 strongly believed that the RTs in School B had sufficient skills to manage students with SEN in the regular classroom but as a result of the large number of students, it posed difficulties. Hence, it was indicated that teaching assistants should be provided for the RTs when managing a large number of students in the regular classroom.

5.1.3. Trained Teachers. Just as in other organizations, schools also need the right number of employees to function properly. Similarly, the participants in this study voiced trained teachers as a perceived support needed for implementing inclusive education. This sub-theme was common across all of the teacher groups in this study. From the responses received from the open-ended item in the survey questionnaire, a total of 25 RTs (9.7\%), 14 STs (20.6\%) and 4 DPs (13.3\%) described that trained teachers were greatly needed for inclusive education.

The RTs in this study did not feel they had the training and expertise needed for inclusive education. One of the reasons according to the RTs was a lack of knowledge and practical experiences.

The narrative of RT1 of this study elaborated on the extent of the knowledge for inclusive education provided during the teacher training, implying a lack of in-depth knowledge on managing different disabilities. For this reason, the need for in-depth knowledge during 
teacher training was evident. Additionally, RT1 also highlighted the fact there were no practical experiences provided during teacher training. Hence, the initial teacher preparation program for RTs needs to include teaching experiences for students with SEN as part of the training. If carefully designed teacher training was provided to the RTs, the need for more trained teachers for inclusive education would be minimized. Another approach to this issue could be providing inservice training. According to the participants, the inservice training conducted as part of a PD session did not suffice. Similar to the experiences of RT1, RT8 also did not receive in-depth knowledge of inclusive education during the initial teacher training program. Additionally, the PD sessions provided by School G were not efficient, as it was limited to a short period of time. Further, enough information was not provided during the short PD sessions.

\subsection{School Supports}

Many of the participants in this study expressed that more support to implement inclusive education was required from the school. Some of the sub-themes that emerged under school supports were support from the school management and further teacher training.

5.2.1. Support from the school management. The teachers in this study articulated their need for more support from the school management. The responses showed that 53 RTs $(20.5 \%)$ and 11 STs (16.2\%) felt that a more efficient support system was needed than the existing supports at the time of this study.

According to RT1, the school management in School $\mathrm{J}$ paid careful attention to the progress of the SEN students and thought of different ways to accommodate the SEN students to maximize their potential. Similarly, RT2 from School H described how supportive the school's management was in providing inclusive education. RT2 surmised the ways in which the school management of School $\mathrm{H}$ provided support to the teachers, like allocating a teacher to oversee all the students with SEN in the school.

Another school where school management was supportive of inclusive education was School G. According to ST3, the school management of School $\mathrm{G}$ tried to provide the material needed for students with SEN and carefully coordinated the learning of these students. Although the school management of schools such as School J, School H and School G provided good support for implementing inclusive education, it was evident from the responses of some of the participants that more support was required from the school management of other schools in this study.

RT3 highlighted that support from the management of School I was mostly required to manage the students in the regular classrooms. It was stated that priority was given to SEN and it was implied that more support staff were required for the regular classroom. RT3's statement indicated a feeling of dissatisfaction or hostility with the phrase "They give priority to SEN", insinuating that the RTs were kind of neglected with regards to needing support. On the contrary, students with SEN should be provided with extra support in inclusive education compared to regular students. As a result, the RTs' tasks would be much easier. It was articulated by RT7 that the school management of School C was not committed to monitoring of the progress of the students with SEN. The only support received from the management according to RT7 was sorting out issues related to the discipline of the students. The statement of RT7 also highlighted the limited commitment of the managers of School $\mathrm{C}$ in supporting teachers during the implementation of inclusive education. Based on these findings, it is evident that the management of School C should pay attention to the students with SEN and plan out the progress of these students by way of close coordination with the teachers. Otherwise, the school would not be providing inclusive education at all, but rather integrating students with SEN in a regular classroom without the required support. This could cause harm both to the regular students and the students with SEN.

5.2.2. Reduced Class Size. The participants in this study also reported that the large size class was difficult for the teachers to manage. The need for reduced class size as a support was reported by $15 \mathrm{RTs}$ (5.8\%), 6 STs $(8.8 \%)$ and 3 DPs $(10.0 \%)$. To cope with the large number of students, the teachers expressed the need for more classrooms to accommodate the students. RT3 from School I explained the difficulty of having too many students in one classroom.

According to RT3, School I faced the problem of having too many students in a regular classroom, since just one regular teacher was monitoring all. In the case of RT3, there were 30 students to be managed along with students with SEN. This also reflects on the absence of an assistant teacher or shadow teacher to help the RTs manage inclusive education in the regular classrooms in School I. Also, the school leaders, such as DP4 (School C) shared the same views of the RTs. As a school leader, DP4 understood that teachers in School C also struggled with the number of students in classrooms. The statement of DP4 also meant that as a school leader being aware of this issue, there was not much that could be done about it. This leads to the decisions of the policymakers in determining the student-teacher ratio in each school.

\section{Teacher Training}

The participants in this study also reported teacher training as a perceived support needed for inclusive education. A total of 55 RTs $(21.2 \%), 11$ STs (16.2\%) and 6 DPs $(20.0 \%)$ reported that teacher training was required in their schools. The teachers revealed that the schools mostly provided further training through PD 
sessions.

According to DP3, School A had identified areas for teacher awareness in inclusive education and conducted more than one session throughout the year. Further, it was also evident that more focus was given to prepare the teachers for the students' IEP.

From the narrative of DP3, it was understood that School A was focused on implementing inclusive education by identifying the teachers' needs and conducting PD sessions according to the requirement of the teachers. This was regardless of the fact that the academic calendar by MoE included only three PD days. The narrative of ST3 described in detail the nature of past PDs held in School C. It was evident that School $\mathrm{C}$ had complied with inclusive policy in preparing teachers to include all students in the regular classroom. Also, as PD focused on curriculum adaptation, ST1 felt that it was a very structured program and highlighted the fact that the school was taking precautions and making progress in providing in-service teacher training. This view was supported with the phrase "the school has taken a good initiative", which implied such practices were not in place previously in School C. Therefore, it was evident that School $\mathrm{C}$ was making efforts to implement the inclusive education policy.

From the statements of the STs and DPs, it was affirmed that the PD sessions were useful and beneficial. Nevertheless, many RTs voiced the ineffectiveness of the one-day PD sessions. This was identified by the RTs of School J, School C and School F, who felt that one-day PD sessions were not helpful. It was evident from the narrative of RT1 that the short sessions conducted as a form of PD were not sufficient. This means that the PD sessions need to be continued consistently throughout the year with more in-depth information as necessary for the RTs. Therefore, the management of School J needs to have a well-structured plan for in-service teacher training.

According to RT7, School C had not conducted many PD sessions based on the aspects of inclusive education. The manner in which a PD session on ASD was conducted was not very effective, as RT7 described that the information was not very clear. This further implies a lack of effective planning of the PD sessions. Another striking fact was that RT7 did not get the opportunity to attend any PD sessions, except for one session in past two years. This is a concern, as all the RTs should have had opportunities for further training to equip them with the skills and knowledge to implement inclusive education in regular classrooms. In contrast, School F provided all of the RTs with the opportunity of PD sessions. Despite this, the sessions did not seem to have an impact. Even though the PD sessions were conducted in School F for all the RTs and STs, it was found not to suit the needs of the RTs. This indicated that School F should conduct a needs analysis to determine the areas of SEN in which the RTs needed more information about the strategies to teach and manage students with SEN in an inclusive classroom and tailor the PD sessions according to the RTs needs.

\section{Discussion}

The main resources needed by the stakeholders in this study were found to be resources such as teachinglearning materials for inclusive teaching, teaching assistants, and trained teachers, and other school support like support from the school management, reduced class size and teacher training.

\subsection{Resources}

It is important for teachers to reach all learners in an inclusive classroom. This could be achieved with sufficient teaching materials to create an interesting and engaging learning environment. The lack of materials and resources being limited in the schools, may impede the successful implementation of inclusive education. By modifying the curriculum and instructional techniques will benefit students with SEN.

Another sub-theme that emerged as a resource in relation to the required support for inclusive education in this study was teaching assistants. From the openended item in the survey questionnaire, the RTs, STs and DPs stated that teaching assistants were required in their schools for inclusive education. From the interviews, the STs and the DPs stated the need for teaching assistants. For example, DP4 from School C described that the support of assistant teachers was required for the RTs. Similarly, ST4 from School B indicated that the RTs could not manage teaching students with SEN and regular students together in the regular classrooms without the help of an assistant teacher. Also, ST4 explained that the RTs would have the knowledge about special needs and how to attend to students with SEN, but due to the large class sizes, the RTs were not able to provide individual attention. These findings reflected on the common issue among all the participants that there were too many students in the regular classes than adequate for the RTs to manage on their own. One possible way to manage the situation would be to control the student-teacher ratio of classes in the inclusive schools. Another way to manage this situation would be to allocate trained teachers as assistant teachers for the RTs. Many studies have highlighted the importance of trained teachers for inclusive education.

Another sub-theme that emerged from the responses of the participants was trained teachers. The interview responses showed that all the STs were trained, however, many RTs in this study narrated their lack of knowledge for inclusive education. Mostly, the RTs reported not having much in-depth knowledge of inclusive education during their initial teacher training and as RT1 explained, there were no face-to-face experiences in teaching students with SEN during their training. According to these responses, teachers could be provided with further knowledge on SEN through PD sessions. These findings signify that the PD sessions should be in-depth, practical and consistent. If 
RTs could be provided with strategies to manage the learning of different ability students, then it would be very helpful. Schools should take the initiative to provide RTs with support such as handbooks and teacher guides, so the RTs could fill in the positions of trained teachers for inclusive education in the schools. It was suggested that teachers should receive inservice training for managing inclusive education through well-structured training and staff development programs [16].

\subsection{School Supports}

The second major theme that emerged from the participants' perceived support needed in the current study was school support. The sub-themes under school support were support from school management, reduced class size and teacher training. Research has indicated that the instructional leadership of school principals plays a crucial part in inclusive school reforms [17]. Thus, school leaders in the inclusive schools of the Maldives need to take an instructional leadership approach when implementing inclusive education. In addition to practicing an instructional leadership style, school principals' need to have positive beliefs about inclusive education and be committed to making inclusive education work in their schools.

Regarding the large class size, it was found that respondents reported dissatisfaction about the class size and being unable to provide individual attention [18]. Studies also show that this can contribute to the teachers developing negative feelings. Many students in a regular classroom could affect the teachers' confidence and autonomy when implementing inclusive education. Hence, a large class size is seen as a barrier to successful inclusion.

Findings of this study indicate that the policymakers should conduct well-planned and continuous professional development (CPD) to enhance the teachers' knowledge and capacity for inclusive education in the Maldives. It was suggested that besides professional support for managing students in the regular schools, support for teachers, assistance in the preparation of learning materials and adaptations, individualized program design support, planning and the evaluation of adaptations and support for the development of intervention programs should be provided [19]. It is of great concern that many of the RTs, the STs and all the DPs in this study perceived teaching materials as a support needed to implement inclusive education. School principals should ensure that suitable support is provided for staff to implement inclusive education. As inclusive education is in the early years of implementation in the Maldives, this finding served as an implication for the policymakers to ensure that the schools are provided with the necessary teaching materials. Additionally, this finding should also be considered as a high priority by the MoE in planning and budgeting for inclusive education in the Maldives.
The findings of this study about the important role of school support in contributing to the implementation process of inclusive education is well-evidenced. Providing school support to teachers is mainly the school administrator's responsibility. The main goal of an administrator is to create strategies for teachers to embrace inclusive education [20]. The school principals' support of inclusion is found to be a significant determining factor in creating effective inclusive schools. Schools with strong administrative support and commitment would be able to cater to more students with diverse needs in a regular education setting. Therefore, the school leaders of inclusive schools in the Maldives should ensure that the necessary support for the staff of their schools is provided for inclusive education to be successful.

\section{Recommendations}

\subsection{Policy makers}

Based on the findings of this study it is important that the policymakers ensure that teaching resources such as alternative texts, braille, large print and other means of assistive technology are provided to schools. Also, it is important to review and ensure that all teacher training courses reflect inclusive education policies, methods, information and teaching strategies for students with SEN. Furthermore, teacher training should be conducted to supply well-trained teachers for inclusive education for all the schools. It is highly important that policymakers review and amend the current policy on teacher allocation for inclusive education to ensure teachers are allocated appropriately, acknowledging the severity of students with SEN in each school. Also, it was evident that more support at the national level should be provided for inclusive education.

\subsection{School Leaders}

This study also identified implications for the school leaders. The school leaders should provide teachers with knowledge of assistive technology, adaptive instruction, differentiated teaching and strategies in managing students with SEN as continued professional development. School leaders should plan and conduct awareness campaigns on the importance and understanding of inclusive education for the parents and school staff, including non- academic staff to instill positive beliefs about inclusion. Also, school leaders should implement strategies to collaborate with schools within the same region to share success stories, combined professional development, share resources and co- teach.

\section{Conclusion}

This study was able to identify the perceived supports needed to implement inclusive education by 
the stakeholders in this study. The two identified themes were resources and school support. The main resources identified by the stakeholders were resources pertaining to the teaching-learning process, such as adapted material for diverse learners like books, puzzles and worksheets. It was also found from the interviews that the materials being used at the time of this study for students with SEN were not differentiated. The other resources identified in this study were teacher assistants and trained teachers. This study found that regular teachers needed teaching assistants to help manage students with SEN in the regular classrooms. Further, it was found that schools in this study needed more teachers trained for inclusive education

\section{References}

[1] Savolainen, H., Engelbrecht, P., Nel, M., \& Malinen, O. (2012). Understanding teachers' attitudes and self- efficacy in inclusive education: Implications for pre- service and inservice teacher education. European Journal of Special Needs Education, 27(1), 51-68.

[2] Holahan, A., \& Costenbader, V. (2000). A comparison of developmental gains for preschool children with disabilities in inclusive and self- contained classrooms. Topics in Early Childhood Special Education, 20, 224- 235.

[3] Kavale, K.,\& Forness, S. (2000). History, rhetoric, and reality; Analysis of the inclusion debate. Remedial and Special Education, 21(5), 279-295.

[4] McCarty, K. (2006). Full Inclusion: The Benefits and Disadvantages of Inclusive Schooling. An Overview. Online Submission, 1-11.

[5] Strain, P.S., Bovey, E.H., Wilson, K., \& Roybal, R. (2009). LEAP preschool: Les- sons learned over 28 years of inclusive services for young children with autism. Young Exceptional Children Monograph Series, 11, 49- 68.

[6] Nishan, F. (2018). Challenges of regular teachers in implementing inclusive education in schools of Maldives. International Journal of Education, Psychology and Counseling, 3(10), 88-102

[7] Naseer, B. (2012). Moving towards inclusion: A case study of one urban school in the Maldives. Universtiy of Canterbury.

[8] Ministry of Education. (2017). School Statistics, 2017. Republic of Maldives. Retrieved from https://www.moe. gov.mv/category/view/14. (Access date: 12 April 2017).

[9] Lyon, G., Vaassen, M., \& Toomey, F.(1989). Teachers' perceptions of their undergraduate and graduate preparation. Teacher Education and Special Education, 12 (4), 164-69.

[10] Burstein, N., Sears, S., Wilcoxen, A., Cabello, B., \& Spagna, M. (2004). Moving toward inclusive practices. Remedial and Special Education, 43(1), 104-116.

[11] Avramidis, E., Bayliss, P., \& Burden, R. (2000). A Survey into Mainstream Teachers' Attitudes Towards the Inclusion of Children with Special Educational Needs in the
Ordinary School in one Local Education Authority. Educational Psychology, 20(2), 191-211.

[12] Pavri, S. \& Hegwer-DiVita, M. (2006). Meeting the social and emotional needs of students with disabilities: The special educators' perspective. Reading \& Writing Quarterly, 22, 139-153.

[13] McClean, W. A. (2007). An investigation into the need for effective leadership mechanisms in the man- agement of a successful inclusive programme in the primary school system.

[14] NCSE. (2013). Supporting Students with Special Educational Needs in Schools, (4), 1-190.

[15] Braun, V. and Clarke, V. (2006) Using thematic analysis in psychology. Qualitative Research in Psychology, 3 (2). pp. $77-101$.

[16] Geldenhuys, J. L., \& Wevers, N. E. J. (2013). Ecological aspects influencing the implementation of inclusive education in mainstream primary schools in the Eastern Cape, South Africa. South African Journal of Education, 33(3), 1-18.

[17] Leithwood, K. (2010). Four key policy questions about parent engagement: Recommen- dations from the evidence, In R. Deslandes (ed.). International perspectives on contexts, communities and evaluated innovative practice (pp. 820). London: Routledge.

[18] Mahoney, C. (2016). Assessing Teachers' Attitudes towards Inclusive Education within an Urban School District in Ireland. Dublin Business School.

[19] Schmidt, Majda \& Vrhovnik, Ksenja. (2015). Attitudes of Teachers Towards the Inclusion of Children With Special Needs in Primary and Secondary Schools. Hrvatska Revija Za Rehabilitacijska Istrazivanja. 51. 16- 30.

[20] Mcmillan, N. M. (2008). Inclusive Education: The Benefits and the Obstacles. Education and Human Development. Education and Human Development Master's Theses. 